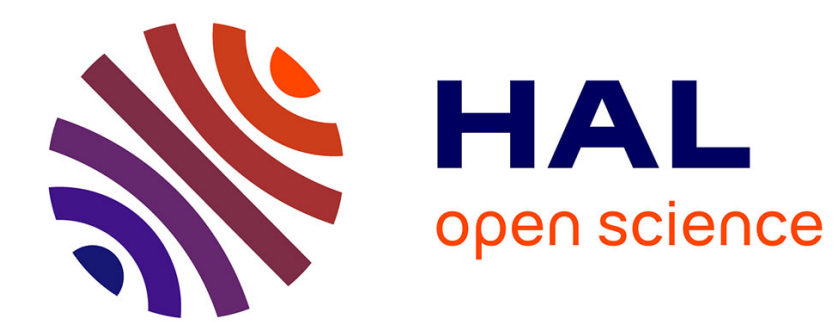

\title{
Prediction-based controller for linear systems with stochastic input delay
}

Sijia Kong, Delphine Bresch-Pietri

\section{To cite this version:}

Sijia Kong, Delphine Bresch-Pietri. Prediction-based controller for linear systems with stochastic input delay. 2021. hal-03270244v1

\section{HAL Id: hal-03270244 \\ https://hal.science/hal-03270244v1}

Preprint submitted on 24 Jun 2021 (v1), last revised 30 Nov 2021 (v3)

HAL is a multi-disciplinary open access archive for the deposit and dissemination of scientific research documents, whether they are published or not. The documents may come from teaching and research institutions in France or abroad, or from public or private research centers.
L'archive ouverte pluridisciplinaire HAL, est destinée au dépôt et à la diffusion de documents scientifiques de niveau recherche, publiés ou non, émanant des établissements d'enseignement et de recherche français ou étrangers, des laboratoires publics ou privés. 


\title{
Prediction-based controller for linear systems with stochastic input delay
}

\author{
SiJia Kong a , Delphine Bresch-Pietri ${ }^{\text {a }}$ \\ ${ }^{a}$ MINES ParisTech, PSL Research University CAS-Centre Automatique et Systèmes, 60 Boulevard Saint Michel 75006 Paris, France
}

\begin{abstract}
We study the stabilization problem of a linear time-invariant system with an unknown stochastic input delay. We propose to robustly compensate for the stochastic delay with a constant time horizon prediction-based controller. We prove the mean-square exponential stabilization of the closed-loop system under a sufficient condition, which requires the range of the delay values to be sufficiently narrow and the constant delay used in the prediction-based controller to be chosen in this range. Numerical simulations illustrate the relevance of this condition and the merits of our control design.
\end{abstract}

Key words: linear systems; delay systems; random delay; backstepping control of distributed parameter systems; prediction-based control; delay systems.

For reviewers' convenience, we have highlighted the main modifications of the paper in blue. The list of these changes is not exhaustive but refers to the major points addressed in the Response.

\section{Introduction}

Time delays are ubiquitous in engineering systems. Especially, the development of communication technology led to the large spread of sophisticated network control systems $[36,41]$. Yet, information transmitted through these networks often suffers from lag [37], data reordering, packets dropouts [11], data corruption or quantization [7]. Such phenomena play a crucial role in the dynamic of vehicular traffic [14]. Indeed, in addition to the driver reaction time, wireless vehicle-to-vehicle communication [42] used to monitor vehicles ahead when beyond the line of sight often introduces substantial communication delays and packet losses $[4,30]$ while transmitting the remote vehicles information. These phenomena can be accounted for by a stochastic delay model (see $[12,13])$.

When a delay affects the input of a dynamical system, prediction-based laws $[1,38]$ are the state-of-the-art control strategy [33]. It was first applied to linear systems with

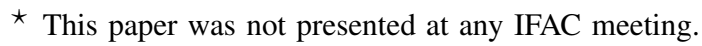

Email addresses: sijia.kong@mines-paristech.fr (SiJia Kong),

delphine.bresch-pietri@mines-paristech.fr (Delphine Bresch-Pietri).
}

constant input delays (see $[25,28]$ ), then extended to handle time-varying delays (see $[2,32]$ ), uncertain input delays or disturbances (see $[26,31]$ ), and nonlinear systems (see $[3,18]$ ). The main ingredient of this class of control law requires calculating a state prediction over a time window of the length of the delay or future value of the delay in the case of a time-varying delay. However, this strategy difficultly translates to the case of unknown future delay variations and even more to stochastic delays.

While a vast number of works $[15,16,20,29,39]$ have investigated the stability or stabilization of Stochastic Delay Differential Equations (SDDEs) in various contexts, only a few works have considered the case where the delay itself is a stochastic variable. Indeed, prediction-based control laws have been applied to linear SDDEs in [6], but the delay itself is assumed to be constant. Up to our knowledge, ones of the few studies to consider the delay as a stochastic variable are [21,22,27,40]. While [40] studies a piecewise constant process and [27] analyzes a deterministic delay term multiplied by a random variable, [21,22] consider stochastic state delays modeled as a Markov process with a finite number of states. The authors then consider each delay value separately, following the so-called technique of probabilistic delay averaging. This constant delay reasoning inspired the core of our analysis methodology.

In this paper, we consider for the first time the problem of prediction-based control of dynamical systems subject to stochastic input delays. We consider linear dynamics and model the delay as a Markov process with a finite number of 
states. We propose to use a prediction-based controller aiming at compensating for this delay. Yet, due to its stochastic nature and the fact that the current delay value is unlikely to be measured, we design a constant-horizon prediction.

This control strategy builds on the delay-robustness results obtained for prediction-based controllers in the deterministic case. Indeed, delay-robust compensation can be achieved not only for a constant time-delay [31], but also for a timevarying one provided that the delay rate remains sufficiently small [3] or using a small-gain approach [17]. In this paper, we extend these results to the stochastic delay case and establish that a constant horizon prediction-based controller guarantees mean-square exponential stabilization of the system provided that the horizon prediction is sufficiently close to the delay values. This is the main contribution of the paper.

This paper is organized as follows. In section 2, we begin with the problem statement and state our main theorem. In section 3, we propose a backstepping transformation to reformulate the system, which establishes the preliminaries to analyze the stability of the closed-loop system in section 4. Simulation results are given in section 5 to illustrate the relevance of the stabilization conditions we formulated.

\section{Notations.}

In the following sections, for a signal $v:(x, t) \in[0,1] \times \mathbb{R} \rightarrow$ $v(x, t) \in \mathbb{R}$, we denote $\|v(t)\|$ its spatial $\mathscr{L}_{2}$-norm

$$
\|v(t)\|=\sqrt{\int_{0}^{1} v(x, t)^{2} d x}
$$

Additionally, for a real matrix $A,|A|$ denotes its Euclidean norm

$$
|A|=\sqrt{\max \left(\lambda\left(A^{T} A\right)\right)}
$$

in which $\lambda$ represents the eigenvalues of the matrix, and $A^{T}$ denotes the transpose of $A$.

$\mathbb{E}[x]$ denotes the expectation of a random variable $x$. For a random signal $x(t)(t \in \mathscr{T} \subset \mathbb{R})$, the conditional expectation of $x(t)$ at the instant $t$ knowing that $x(s)=x_{0}$ at the instant $s \leq t$ is denoted $\mathbb{E}_{\left[s, x_{0}\right]}[x(t)]$.

Finally, $e_{i} \in \mathbb{R}^{r}\left(r \in \mathbb{N}_{+}\right.$and $\left.i \in\{1, \ldots, r\}\right)$ denotes the $i^{t h}$ unit vector, that is, $e_{1}=\left(\begin{array}{llll}1 & 0 & \cdots & 0\end{array}\right)^{T}, e_{2}=\left(\begin{array}{llll}0 & 1 & \cdots & 0\end{array}\right)^{T}$, $\ldots, e_{r}=\left(\begin{array}{llll}0 & 0 & \cdots & 1\end{array}\right)$.

\section{Problem Statement and Main Result}

We consider the following controllable linear dynamics

$$
\dot{X}(t)=A X(t)+B U(t-D(t))
$$

in which the $\mathbb{R}^{n}$-valued random variable $X$ and $U \in \mathbb{R}$ are the state and control input, respectively. The stochastic delay $D$ is a Markov process with the following properties:

(1) $D(t) \in\left\{D_{i}, i \in\{1, \ldots, r\}\right\}, r \in \mathbb{N}$ with $0<\underline{D} \leq D_{1}<D_{2}<$ $\ldots<D_{r} \leq \bar{D}$.

(2) The transition probabilities $P_{i j}\left(t_{1}, t_{2}\right)$, which quantify the probability to switch from $D_{i}$ at time $t_{1}$ to $D_{j}$ at time $t_{2}$ $\left((i, j) \in\{1, \ldots, r\}^{2}, t_{2} \geq t_{1} \geq 0\right)$, are differentiable functions $P_{i j}: \mathbb{R}^{2} \rightarrow[0,1]$ satisfying

$$
\sum_{j=1}^{r} P_{i j}\left(t_{1}, t_{2}\right)=1, \quad\left(0 \leq t_{1} \leq t_{2}\right)
$$

We consider the following constant time horizon predictionbased control law

$$
U(t)=K\left[e^{A D_{0}} X(t)+\int_{t-D_{0}}^{t} e^{A(t-s)} B U(s) d s\right]
$$

in which $K$ is a feedback gain such that $A+B K$ is Hurwitz, and $D_{0} \in[\underline{D}, \bar{D}]$ is constant.

Exact compensation of the non-constant delay in (3) requires the function $\phi: t \rightarrow t-D(t)$ to be invertible in order to define the feedback law $U(t)=K X\left(\phi^{-1}(t)\right)$. However, in the case of a stochastic delay, the function has no reason to be invertible. In addition, even if it were, the computation of $\phi^{-1}(t)$ would require to know future realizations of the delay which is impossible in practice.

For these reasons, we therefore propose to use the constant horizon prediction-based controller (5). Note that, if the time lag $D$ was constant and equal to $D_{0}$, the control law would then correspond to the exact prediction of the state $X$ over a time window of $D_{0}$ units. Consistently, we now formulate the main result of the paper which states that robust compensation is achieved if the time delay remains sufficiently close to $D_{0}$.

Theorem 1 Consider the closed-loop system consisting of the system (3) and the control law (5). Then, there exists a positive constant $\varepsilon^{\star}(K)$ such that, if

$$
\left|D_{0}-D_{j}\right| \leq \varepsilon^{\star}(K), \quad j \in\{1, \ldots, r\}
$$

there exist positive constants $R$ and $\gamma$ such that

$$
\mathbb{E}_{[0, \Upsilon(0)]}[\Upsilon(t)] \leq R \Upsilon(0) e^{-\gamma t}
$$

with

$$
\Upsilon(t)=|X(t)|^{2}+\int_{t-\bar{D}-D_{0}}^{t} U(s)^{2} d s
$$

The prediction-based control law (5) would exactly compensate for the input delay with a constant time lag equal to $D_{0}$. In other words, if $D(t)=D_{0}$, applying the variation of 
constant formula, it would follow that $U(t)=K X\left(t+D_{0}\right)$ and that, after $D_{0}$ units of time, the corresponding closedloop dynamics would be $\dot{X}=(A+B K) X$ which is exponentially stable. Condition (6) guarantees that the prediction performed in (5) remains sufficiently accurate in the case of a stochastic delay. In details, (6) requires the sequence of the random delay $\mathbf{D}=\left(D_{1} \ldots D_{i} \ldots D_{r}\right)_{r \in \mathbb{N}}^{T}$ to be limited in a vicinity $\varepsilon^{*}$ of the constant $D_{0}$.

Note that this result is consistent with the delay-robustness results obtained in the deterministic delay case. Indeed, [3, 23 ] provide a similar robust compensation result for a timedifferentiable delay function, under the assumptions that both the range of variation of the delay and its variation rate is sufficiently limited. A similar result was obtained in [17] but through a small-gain approach enabling to avoid restricting the delay rate. Finally, as the delay process under consideration only takes a finite number of values, it is worth noticing that similar robustness properties were also obtained in a discrete-time context in [8] where the prediction is approximated to respect causality. Hence, this theorem falls within this framework and extends it to the stochastic context.

Finally, note that the limit $\varepsilon^{\star}$ depends on the feedback gain $K$, the choice of which is likely to play a crucial role in practice. However, capturing this dependence is a complex task from a Lyapunov stability point of view and would require additional studies.

We now provide the proof of this theorem in the following sections.

\section{PDE Representation of the Delay and Backstepping Transformation}

First, to represent the control input which is subject to a stochastic delay, we define a distributed actuator vector as, for $x \in[0,1], \mathbf{v}(x, t)=\left(v_{1}(x, t) \ldots v_{k}(x, t) \ldots v_{r}(x, t)\right)^{T}$ with $v_{k}(x, t)=U\left(t+D_{k}(x-1)\right)$. This enables to rewrite (3) as

$$
\left\{\begin{aligned}
\dot{X}(t) & =A X(t)+B \delta(t)^{T} \mathbf{v}(0, t) \\
\Lambda_{D} \mathbf{v}_{t}(x, t) & =\mathbf{v}_{x}(x, t) \\
\mathbf{v}(1, t) & =\mathbf{1} U(t)
\end{aligned}\right.
$$

in which $\Lambda_{D}=\operatorname{diag}\left(D_{1}, \ldots, D_{r}\right), \mathbf{1}$ is a $r$-by-1 all-ones vector and $\delta(t) \in \mathbb{R}^{r}$ is such that, if $D(t)=D_{j}$,

$$
\delta_{i}(t)= \begin{cases}1 & \text { if } i=j \\ 0 & \text { otherwise }\end{cases}
$$

Hence, $\delta(t)$ is a Markov process with the same transition probabilities as the process $D(t)$, but with the finite number of states $\left(e_{i}\right)$ instead of $\left(D_{i}\right)$. In the sequel, $\delta(t)$ and $D(t)$ will thus be equivalently used.
Now, we introduce $\hat{v}(x, t)$ to represent the control input $U(t)$ within the interval $\left[t-D_{0}, t\right]$, and the corresponding input estimation error $\tilde{\mathbf{v}}(x, t)$ defined as

$$
\left\{\begin{array}{l}
\hat{v}(x, t)=U\left(t+D_{0}(x-1)\right) \\
\tilde{\mathbf{v}}(x, t)=\mathbf{v}(x, t)-\mathbf{1} \hat{v}(x, t)
\end{array}\right.
$$

Then, the extended state $(X(t), \hat{v}(x, t), \tilde{\mathbf{v}}(x, t))$ satisfies

$$
\left\{\begin{aligned}
\dot{X}(t) & =A X(t)+B \hat{v}(0, t)+B \delta(t)^{T} \tilde{\mathbf{v}}(0, t) \\
D_{0} \hat{v}_{t}(x, t) & =\hat{v}_{x}(x, t) \\
\hat{v}(1, t) & =U(t) \\
\Lambda_{D} \tilde{\mathbf{v}}_{t}(x, t) & =\tilde{\mathbf{v}}_{x}-\Sigma_{D} \hat{v}_{x} \\
\tilde{\mathbf{v}}(1, t) & =\mathbf{0}
\end{aligned}\right.
$$

in which $\Sigma_{D}=\left(\frac{D_{1}-D_{0}}{D_{0}}, \ldots, \frac{D_{r}-D_{0}}{D_{0}}\right)^{T}$ and $\mathbf{0}$ is a $r$-by-1 allzeros vector.

Besides, to ease the stability analysis, we also introduce another actuator $\mu(x, t)=U\left(t-D_{0}+\bar{D}(x-1)\right)$ to describe the history of the input on a longer time window $\left[t-\bar{D}-D_{0}, t-D_{0}\right]$. Correspondingly, we extend the dynamic to $(X(t), \hat{v}(x, t), \tilde{\mathbf{v}}(x, t), \mu(x, t))$ satisfying

$$
\left\{\begin{aligned}
\dot{X}(t) & =A X(t)+B \hat{v}(0, t)+B \delta(t)^{T} \tilde{\mathbf{v}}(0, t) \\
D_{0} \hat{v}_{t}(x, t) & =\hat{v}_{x}(x, t) \\
\hat{v}(1, t) & =U(t) \\
\Lambda_{D} \tilde{\mathbf{v}}_{t}(x, t) & =\tilde{\mathbf{v}}_{x}-\Sigma_{D} \hat{v}_{x} \\
\tilde{\mathbf{v}}(1, t) & =\mathbf{0} \\
\bar{D} \mu_{t}(x, t) & =\mu_{x}(x, t) \\
\mu(1, t) & =\hat{v}(0, t)
\end{aligned}\right.
$$

In other words, $\hat{v}$ now cascades into the transport PDE satisfied by $\mu$. Finally, in view of stability analysis, we introduce the backstepping transformation (see [24])

$$
\begin{aligned}
w(x, t)= & \hat{v}(x, t)-K e^{A D_{0} x} X(t) \\
& -D_{0} \int_{0}^{x} K e^{A D_{0}(x-y)} B \hat{v}(y, t) d y
\end{aligned}
$$

Lemma 2 The backstepping transformation (14), jointly with the control law (5), transform the plant (13) into the target system $(X(t), w(x, t), \tilde{\mathbf{v}}(x, t), \mu(x, t))$

$$
\left\{\begin{aligned}
\dot{X}(t) & =(A+B K) X(t)+B \delta(t)^{T} \tilde{\mathbf{v}}(0, t)+B w(0, t) \\
D_{0} w_{t}(x, t) & =w_{x}(x, t)-D_{0} K e^{A D_{0} x} B \delta(t)^{T} \tilde{\mathbf{v}}(0, t) \\
w(1, t) & =0 \\
\Lambda_{D} \tilde{\mathbf{v}}_{t}(x, t) & =\tilde{\mathbf{v}}_{x}-\Sigma_{D} h\left(t+D_{0}(x-1)\right) \\
\tilde{\mathbf{v}}(1, t) & =\mathbf{0} \\
\bar{D} \mu_{t}(x, t) & =\mu_{x}(x, t) \\
\mu(1, t) & =K X(t)+w(0, t)
\end{aligned}\right.
$$


in which, $h$ is defined for $t \geq 0$ as

$$
\begin{aligned}
& h(t)=D_{0} K\left[(A+B K) e^{A D_{0}} X(t)+e^{A D_{0}} B \delta(t)^{T} \tilde{\mathbf{v}}(0, t)\right. \\
& +D_{0}(A+B K) \int_{0}^{1} e^{A D_{0}(1-x)} B\left(w(x, t)+K e^{(A+B K) D_{0} x} X(t)\right. \\
& \left.\left.+\int_{0}^{x} K D_{0} e^{(A+B K) D_{0}(x-y)} B w(y, t) d y\right) d x\right]
\end{aligned}
$$

PROOF. The space-derivative of the backstepping transformation (14) can be written as

$$
\begin{aligned}
w_{x}(x, t)= & \hat{v}_{x}(x, t)-K A D_{0} e^{A D_{0} x} X(t)-D_{0} K B \hat{v}(x, t) \\
& -D_{0} \int_{0}^{x} K A D_{0} e^{A D_{0}(x-y)} B \hat{v}(y, t) d y
\end{aligned}
$$

Besides, the time-derivative of (14) reads

$$
\begin{aligned}
w_{t}(x, t)= & \hat{v}_{t}(x, t)-D_{0} \int_{0}^{x} K e^{A D_{0}(x-y)} B \hat{v}_{t}(y, t) d y \\
& -K e^{A D_{0} x} A X(t)-K e^{A D_{0} x} B \hat{v}(0, t) \\
& -K e^{A D_{0} x} B \delta(t)^{T} \tilde{\mathbf{v}}(0, t)
\end{aligned}
$$

From (14), (17) and (18) with an integration by parts, we obtain the last two equations in (15). Finally, from the definition of $\tilde{\mathbf{v}}$ and $\hat{v}$ in (11), one can observe that $h\left(t+D_{0}(x-1)\right)=$ $\hat{v}_{x}(x, t)=D_{0} \dot{U}\left(t+D_{0}(x-1)\right)$ which gives the desired expression of $h(t)$ for $t \geq 0$, taking a time-derivative of (5) and using the inverse backstepping transformation of (14), which is

$$
\begin{aligned}
\hat{v}(x, t)= & w(x, t)+K e^{(A+B K) D_{0} x} X(t) \\
& +\int_{0}^{x} K D_{0} e^{(A+B K) D_{0}(x-y)} B w(y, t) d y
\end{aligned}
$$

We are now ready to carry out the stability analysis.

\section{Lyapunov Stability Analysis}

\subsection{Preliminaries}

Let us define the state of the target system (15) as $\Psi=$ $(X, w, \tilde{\mathbf{v}}, \mu) \in\left\{\Psi \in \mathbb{R}^{n} \times \mathscr{L}_{2}([0,1], \mathbb{R}) \times \mathscr{L}_{2}\left([0,1], \mathbb{R}^{r}\right) \times\right.$ $\mathscr{L}_{2}([0,1], \mathbb{R}) \mid \Psi_{2}(1)=\Psi_{3}(1)=\Psi_{4}(1)-K \Psi_{1}-\Psi_{2}(0)=$ $0\} \triangleq \mathscr{D}_{\Psi}$. Note that (15) was reformulated as a dynamical system involving a random parameter, as studied in [19] or [9]. However, the results presented in [9] on the existence of solutions consider a more complex stochastic framework. This is why, for the sake of self-containedness, we now formulate a well-posedness result.
Following [19], by a weak solution to the closed-loop system (3) and (5), we refer to a $\mathbb{R}^{n} \times \mathscr{L}_{2}([-\bar{D}, 0], \mathbb{R}) \times \mathbb{R}$-valued random variable $\left(X\left(X_{0}, t\right), U_{t}\left(U_{0}, \cdot\right), D(t)\right)$, the realizations of which satisfy an integral form of (3) and (5).

Similarly, by a weak solution to (15), we refer to a $\mathscr{D}_{\Psi} \times \mathbb{R}$ valued random variable $\left(X\left(X_{0}, t\right), w\left(w_{0}, \cdot, t\right), \tilde{\mathbf{v}}\left(\tilde{\mathbf{v}}_{0}, \cdot, t\right)\right.$, $\left.\mu\left(\mu_{0}, \cdot, t\right), D(t)\right)$, the realizations of which satisfy a weak formulation of (15), that is, with respect to test functions for the transport PDEs and under an integral form for the ODE.

Lemma 3 For every initial condition $\left(X_{0}, U_{0}\right) \in \mathbb{R}^{n} \times$ $\mathscr{L}_{2}([-\bar{D}, 0], \mathbb{R})$, the closed-loop system consisting of (3) and the control law (5) has a unique weak solution such that

$$
X(t)=e^{A t} X(0)+\int_{0}^{t} e^{A(t-s)} B U(s-D(s)) d s
$$

Consequently, for each initial condition in $\mathscr{D}_{\Psi}$, the target system (15) also has a unique weak solution.

PROOF. We first focus on the existence of a solution. Notice that, for $X$ defined in (20), performing an integration by parts,

$$
\begin{aligned}
& X(0)+\int_{0}^{t}(A X(s)+B U(s-D(s))) d s \\
= & X(0)+\int_{0}^{t} B U(s-D(s)) d s+\int_{0}^{t} A e^{A s} X(0) d s \\
& +\int_{0}^{t}\left(\int_{0}^{s} A e^{A(s-\xi)} d s\right) B U(\xi-D(\xi)) d \xi \\
= & e^{A t} X(0)+\int_{0}^{t} e^{A(t-\xi)} B U(\xi-D(\xi)) d \xi=X(t)
\end{aligned}
$$

which corresponds to an integral form of (3). Observe that, as $U_{0} \in \mathscr{L}_{2}([-\bar{D}, 0], \mathbb{R})$, one can prove that both $X$ and $U$, as defined through (20) and (5) remain bounded for positive times, by an iterative argument over time intervals of length $D$ and the use of Cauchy-Schwarz theorem.Thus, one can apply Lebesgue's dominated convergence theorem to prove that the integral in (20) is well-defined. Consequently, (20) and (5) define a weak solution to the closed-loop system.

Secondly, we prove the uniqueness of this solution. Suppose that there exist two different solutions $\left(X_{1}, U_{1}\right)$ and $\left(X_{2}, U_{2}\right)$ for a given initial condition. It then holds

$$
\left\{\begin{aligned}
\left(\dot{X}_{1}-\dot{X}_{2}\right)(t)= & A\left(X_{1}-X_{2}\right) \\
& +B\left(U_{1}(t-D(t))-U_{2}(t-D(t))\right) \\
\left(X_{1}-X_{2}\right)(0)= & 0
\end{aligned}\right.
$$


with $U_{1}=U_{2}$ for $t<0$, and for $t \geq 0$

$$
\left\{\begin{array}{l}
U_{1}(t)=K\left(e^{A D_{0}} X_{1}(t)+\int_{t-D_{0}}^{t} e^{A(t-s)} B U_{1}(s) d s\right) \\
U_{2}(t)=K\left(e^{A D_{0}} X_{2}(t)+\int_{t-D_{0}}^{t} e^{A(t-s)} B U_{2}(s) d s\right)
\end{array}\right.
$$

For any delay realization, it thus holds that $U_{1}(t-D(t))=$ $U_{2}(t-D(t))$ for $t \in[0, \underline{D}]$, which, in turns, gives $X_{1}(t)=$ $X_{2}(t)$ for $t \in[0, \underline{D}]$. Iterating on intervals of length $\underline{D}$, we then obtain $X_{1}=X_{2}$ and $U_{1}=U_{2}$ for $t \in \mathbb{R}_{+}$.

Let us now observe that the well-posedness of the closedloop system (3) and (5) implies the one of (9) and (5) by equivalence. The one of (13) and (5) and thus of (15) by backstepping transformation then follow.

From Lemma 3, $(\Psi, \delta)$ thus defines a continuous-time Markov process and we can therefore introduce the following elements for stability analysis.

In the sequel, we consider the following Lyapunov functional candidate

$$
\begin{aligned}
V(\Psi)= & X^{T} P X+b D_{0} \int_{0}^{1}(1+x) w(x)^{2} d x \\
& +c \sum_{l=1}^{r} \int_{0}^{1}(1+x)\left(\left(e_{l} \cdot \mathbf{D}\right)^{T} \tilde{\mathbf{v}}(x)\right)^{2} d x \\
& +d \bar{D} \int_{0}^{1}(1+x) \mu(x)^{2} d x
\end{aligned}
$$

with $b, c, d>0, P$ the symmetric positive definite solution of the equation $P(A+B K)+(A+B K)^{T} P=-Q$, for a given symmetric positive definite matrix $Q$, and $\mathbf{D}=\left(D_{1} \ldots D_{i} \ldots D_{r}\right)^{T}$ and where $\cdot$ denotes the Hadamard multiplication.

As the functional $V$ is not differentiable with respect to time $t$ when evaluated at $\Psi(t)$, we introduce the infinitesimal generator $L$ (see [22] and [20]) as

$$
L V(\Psi(t))=\limsup _{\Delta t \rightarrow 0^{+}} \frac{1}{\Delta t}\left(\mathbb{E}_{[t, \Psi(t)]}[V(\Psi(t+\Delta t))]-V(\Psi(t))\right)
$$

We also define $L_{j}$, the infinitesimal generator of the Markov process $\Psi$ obtained by fixing the value $\delta(t)=e_{j}$, as

$$
L_{j} V(\Psi)=\frac{d V}{d \Psi}(\Psi) f_{j}(\Psi)
$$

in which $f_{j}$ denotes the operator corresponding to the dynamics of the target system (15) with the fixed value $\delta(t)=$ $e_{j}$, that is, for $\Psi=(X, w, \tilde{\mathbf{v}}, \mu)$,

$$
f_{j}(\Psi)(x)=\left(\begin{array}{c}
(A+B K) X+B e_{j}^{T} \tilde{\mathbf{v}}(0)+B w(0) \\
\frac{1}{D_{0}}\left[w_{x}(x)-D_{0} K e^{A D_{0} x} B e_{j}^{T} \tilde{\mathbf{v}}(0)\right] \\
\Lambda_{D}^{-1}\left[\tilde{\mathbf{v}}_{x}(x)-\Sigma_{D} h\left(\cdot+D_{0}(x-1)\right)\right] \\
\frac{1}{D} \mu_{x}(x)
\end{array}\right)
$$

defined on $\mathscr{D}_{\Psi}$.

For the sake of conciseness, in the sequel, we denote $V(t)$, $L V(t)$ and $L_{j} V(t)$, for short, instead of $V(\Psi(t)), L V(\Psi(t))$ and $L_{j} V(\Psi(t))$ respectively.

It is worth noticing that, due to the fact that $V$ does not depend explicitly on $\delta$ and (4), the infinitesimal generators are related as follows

$$
\begin{aligned}
L V(t) & =\sum_{j=1}^{r} P_{i j}(0, t) \frac{d V}{d \Psi}(\Psi(t)) f_{j}(\Psi(t))+\sum_{j=1}^{r} \frac{\partial P_{i j}}{\partial t}(0, t) V(t) \\
& =\sum_{j=1}^{r} P_{i j}(0, t) L_{j} V(t)
\end{aligned}
$$

Therefore, in view of stability analysis, as a first step, one can focus on the derivative of the Lyapunov functional evaluated for a dynamic with a fixed delay, that is, $L_{j} V$. This is the approach we follow in the sequel.

\subsection{Lyapunov analysis}

Lemma 4 Assume there exist a positive constant $\varepsilon$ such that

$$
\left|D_{0}-D_{j}\right| \leq \varepsilon, \quad j \in\{1, \ldots, r\}
$$

Then, there exist $b, c, d, \eta \in \mathbb{R}_{+}^{*}$ which are independent of $\varepsilon$ such that the Lyapunov functional $V$ defined in (24) satisfies

$$
L V(t) \leq-(\eta-g(\varepsilon)) V(t), \quad t \geq \bar{D}
$$

with the function $g: \mathbb{R}_{+} \rightarrow \mathbb{R}_{+}$satisfying $\lim _{\mathcal{\varepsilon} \rightarrow 0} g(\varepsilon)=0$.

PROOF. Taking a derivative of (24) and applying integra- 
tions by parts and Young's inequality, we obtain

$$
\begin{aligned}
& \frac{d V}{d \Psi}(\Psi) f_{j}(\Psi) \\
= & -X(t)^{T} Q X(t)+2 X(t)^{T} P B\left[w(0, t)+\tilde{v}_{j}(0, t)\right] \\
& +2 b \int_{0}^{1}(1+x) w(x, t)\left[w_{x}(x, t)-D_{0} K e^{A D_{0} x}\right. \\
& \left.\times B \tilde{v}_{j}(0, t)\right] d x+2 c \sum_{l=1}^{r} \int_{0}^{1}(1+x) \tilde{v}_{l}(x, t)\left[\tilde{v}_{l x}(x, t)\right. \\
& \left.+\left(1-\frac{D_{l}}{D_{0}}\right) h\left(t+D_{0}(x-1)\right)\right] d x \\
& +2 d \int_{0}^{1}(1+x) \mu(x, t) \mu_{x}(x, t) d x \\
\leq & -\left(\frac{\min (\lambda(Q))}{2}-4 d|K|^{2}\right)|X(t)|^{2}-d\|\mu(t)\|^{2} \\
& -b\left(1-2 D_{0}|K||B| e^{|A| D_{0}} \gamma_{1}\right)\|w(t)\|^{2} \\
& -c \sum_{l=1}^{r}\left(1-\frac{2}{D_{0}}\left|D_{0}-D_{l}\right| \gamma_{2}\right)\left\|\tilde{v}_{l}(t)\right\|^{2} \\
& -\left(b-4 d-\frac{4|P B|^{2}}{\min (\lambda(Q))}\right) w(0, t)^{2}-\left(c-\frac{4|P B|^{2}}{\min (\lambda(Q))}\right. \\
& \left.-2 b D_{0}|K||B| e^{|A| D_{0}} \frac{1}{\gamma_{1}}\right) \tilde{v}_{j}(0, t)^{2}-c \sum_{l \neq j} \tilde{v}_{l}(0, t)^{2}-d \mu(0, t)^{2} \\
& +\frac{2 c}{D_{0}} \sum_{l=1}^{r}\left|D_{0}-D_{l}\right| \frac{1}{\gamma_{2}}\left\|h\left(t+D_{0}(x-1)\right)\right\|^{2}
\end{aligned}
$$

for any $\gamma_{1}, \gamma_{2} \geq 0$.

Therefore, applying (29) and Lemma 6 given in Appendix, one gets for $t \geq \bar{D}$,

$$
\begin{aligned}
& L V(t) \\
= & \sum_{j=1}^{r} P_{i j}(0, t) \frac{d V}{d \Psi}(\Psi) f_{j}(\Psi) \\
\leq & -\left(\frac{\min (\lambda(Q))}{2}-4 d|K|^{2}\right)|X(t)|^{2} \\
& -b\left(1-2 D_{0}|K||B| e^{|A| D_{0}} \gamma_{1}\right)\|w(t)\|^{2} \\
& -c \sum_{l=1}^{r}\left(1-\frac{2}{D_{0}}\left|D_{0}-D_{l}\right| \gamma_{2}\right)\left\|\tilde{v}_{l}(t)\right\|^{2}-d\|\mu(t)\|^{2} \\
& -\left(b-4 d-\frac{4|P B|^{2}}{\min (\lambda(Q))}\right) w(0, t)^{2}-\left(c-\frac{4|P B|^{2}}{\min (\lambda(Q))}\right. \\
& \left.-2 b D_{0}|K||B| e^{|A| D_{0}} \frac{1}{\gamma_{1}}\right) \sum_{j=1}^{r} P_{i j}(0, t) \tilde{v}_{j}(0, t)^{2} \\
& -c \sum_{j=1}^{r} P_{i j}(0, t) \sum_{l \neq j} \tilde{v}_{l}(0, t)^{2}-d \mu(0, t)^{2} \\
& +\frac{2 c r \varepsilon}{D_{0}} \frac{1}{\gamma_{2}} M V(t)
\end{aligned}
$$

in which the positive constant $M$ does not depend on $\varepsilon$ and is defined in Lemma 6.

Observing that $D_{0} \in[\underline{D}, \bar{D}]$, let us choose $\left(b, c, d, \gamma_{1}, \gamma_{2}\right) \in$ $\left(\mathbb{R}_{+}^{*}\right)^{5}$ as follows

(a) $d<\frac{\min (\lambda(Q))}{8|K|^{2}}$

(b) $b \geq 4 d+\frac{4|P B|^{2}}{\min (\lambda(Q))}$,

(c) $\gamma_{1}<\frac{1}{2 \bar{D}|K| e^{|A| \bar{D}}|B|}$,

(d) $\gamma_{2}<\frac{1}{4} \min \left\{\left(1-\frac{D_{1}}{\bar{D}}\right)^{-1},\left(\frac{D_{r}}{\underline{D}}-1\right)^{-1}\right\}$,

(5) $c \geq \frac{4|P B|^{2}}{\min (\lambda(Q))}+2 b \bar{D}|K||B| e^{|A| \bar{D}} \frac{1}{\gamma_{1}}$.

From (32), one then obtains (30) with the well-defined constant $\eta=\min \left\{\frac{\min (\lambda(Q))-8 d|K|^{2}}{2 \max (\lambda(P))}, \frac{1-2 \bar{D}|K||B| e^{|A| \bar{D}} \gamma_{1}}{2 \bar{D}}, \frac{1}{4 D_{r}}, \frac{1}{2 \bar{D}}\right\}$, and the function

$$
g(\varepsilon)=\frac{2 c r}{D_{0}} \frac{1}{\gamma_{2}} M \varepsilon
$$

which satisfies $\lim _{\mathcal{E} \rightarrow 0} g(\varepsilon)=0$.

\subsection{Proof of Theorem 1}

Firstly, as $\lim _{\varepsilon \rightarrow 0} g(\varepsilon)=0$, there exists $\varepsilon^{*}>0$ such that $\eta-$ $g(\varepsilon)=\gamma>0$ for $\varepsilon<\varepsilon^{*}$. Therefore, according to Dynkin's formula [10, Theorem 5.1, p. 133], from (30), one obtains for $\varepsilon<\varepsilon^{*}$

$\mathbb{E}_{(0, \Psi(0))}[V(t)]-\mathbb{E}_{(0, \Psi(0))}[V(\bar{D})] \leq \mathbb{E}_{(0, \Psi(0))}\left[\int_{\bar{D}}^{t}-\gamma V(s) d s\right]$

Consequently, applying Gronwall's inequality to (34)

$$
\mathbb{E}_{(0, \Psi(0))}[V(t)] \leq \mathbb{E}_{(0, \Psi(0))}[V(\bar{D})] e^{-\gamma(t-\bar{D})}
$$

Noticing that $V$ and $\Upsilon$ are equivalent, that is, that there exist positive constants $q_{1}$ and $q_{2}$ such that for $\forall t \geq 0, q_{1} V(t) \leq$ $\Upsilon(t) \leq q_{2} V(t)$ (see [23]), it thus follows that $\mathbb{E}_{(0, \Upsilon(0))}[\Upsilon(t)] \leq$ $\frac{q_{2}}{q_{1}} \mathbb{E}_{(0, \Upsilon(0))} \Upsilon(\bar{D}) e^{-\gamma(t-\bar{D})}$.

From the definition of $\Upsilon$ in (8), one deduces that there exists a constant $R_{0}$ such that

$$
\Upsilon(t) \leq R_{0} \Upsilon(0), \quad t \in[0, \bar{D}]
$$

(see Lemma 5 in the appendix for a proof of this property). Finally, the function $\Upsilon$ thus satisfies $\mathbb{E}_{(0, \Upsilon(0))}[\Upsilon(t)] \leq$ $\frac{q_{2}}{q_{1}} R_{0} \Upsilon(0) e^{-\gamma(t-\bar{D})}$, Theorem 1 is then proved with $R=\frac{q_{2}}{q_{1}} R_{0} e^{\gamma \bar{D}}$. 


\section{Simulations}

To illustrate Theorem 1 and in particular the role played by the condition (6), we consider the following toy example

$$
\dot{X}(t)=\left[\begin{array}{cc}
0 & 1 \\
-1 & 1
\end{array}\right] X(t)+\left[\begin{array}{l}
0 \\
1
\end{array}\right] U(t-D(t))
$$

The control law (5) is applied with the feedback gain $K=-\left[\begin{array}{ll}1 & 2\end{array}\right]$ resulting in conjugate closed-loop eigenvalues $\lambda(A+B K)=\{-0.5000+1.3229 i,-0.5000-1.3229 i\}$. The initial conditions are chosen as $X(0)=\left[\begin{array}{cc}1 & 0\end{array}\right]^{T}$ and $U(t)=0$, for $t \leq 0$. The integral in (5) is discretized using a trapezoïdal scheme. Finally, the simulations are carried out with a discrete-time solver in Matlab-Simulink and a sampling time $\Delta t=0.01 \mathrm{~s}$.

We consider 5 different delay values $\left(D_{1}, D_{2}, D_{3}, D_{4}, D_{5}\right)=$ $(0.5,0.75,1,1.25,1.5)$. Besides, the initial transition probabilities are taken as $P_{i j}(0,0)=0.4985(i \in\{1, \ldots, 5\}, j=$ $\{3,4\})$ and $P_{i j}(0,0)=0.001(i \in\{1, \ldots, 5\}, j=\{1,2,5\})$, which means that the delay values are initially concentrated in $D_{3}$ and $D_{4}$.

In addition, we introduce the following Kolmogorov equation $[21,34,35]$ to describe the time-evolution of the transition probabilities

$$
\begin{aligned}
\frac{\partial P_{i j}(s, t)}{\partial t} & =-c_{j}(t) P_{i j}(s, t)+\sum_{k=1}^{r} P_{i k}(s, t) v_{k j}(t), s<t \\
P_{i j}(s, s) & =1, \quad \forall i=j \\
P_{i j}(s, s) & =0, \quad \forall i \neq j
\end{aligned}
$$

in which $v_{i j}$ and $c_{j}=\sum_{k=1}^{r} v_{j k}$ are positive-valued functions such that $v_{i i}(t)=0$.

In details, $v_{i j} \Delta t$ is approximately the probability of transition from $D_{i}$ to $D_{j}$ on the interval $[t, t+\Delta t)$. Similarly, $1-c_{j}(t) \Delta t$ represents somehow the probability of staying at $D_{j}$ during the time interval $[t, t+\Delta t)$.

Here, we choose for simulation the transition rates $v_{i j}$ as

$$
\begin{aligned}
v(t) & =\left(\left\{v_{i j}(t)\right\}\right)_{1 \leq i, j \leq r} \\
& =0.03\left(\begin{array}{ccccc}
0 & e^{-10 t} & e^{-10 t} & e^{-10 t} & e^{-10 t} \\
e^{-10 t} & 0 & \frac{1}{2}-e^{-10 t} & \frac{1}{2}-e^{-10 t} & e^{-10 t} \\
e^{-10 t} & 1-3 e^{-10 t} & 0 & e^{-10 t} & e^{-10 t} \\
e^{-10 t} & 1-3 e^{-10 t} & e^{-10 t} & 0 & e^{-10 t} \\
e^{-10 t} & e^{-10 t} & e^{-10 t} & e^{-10 t} & 0
\end{array}\right)
\end{aligned}
$$

The delay values will therefore gradually evolve towards a uniform distribution among the delay values $D_{2}, D_{3}$ and $D_{4}$.
Firstly, we pick $D_{0}=1$. This results in a value $\varepsilon=$ $\max _{j=1, \ldots, r}\left|D_{0}-D_{j}\right|=0.5$. Fig. 1 represents the results obtained for Monte-Carlo simulations of 100 trials. One can observe that the resulting mean value of the state, which approximates the expectation of Theorem 1, indeed converges to the origin.

On the other hand, the choice of a larger prediction horizon $D_{0}=1.25$ (corresponding to the larger value $\varepsilon=0.75$ ) results into a diverging behaviour pictured in Fig. 2. This confirms that the choice of prediction horizon $D_{0}$ should be restricted in a sufficiently small range to guarantee the stability of the dynamics, on average.

It can be seen from the graph of the time lag change (Fig.1.a) that the delay, which originally takes mainly its value among $D_{3}$ and $D_{4}$, becomes gradually evenly distributed between $D_{2}, D_{3}$ and $D_{4}$. This change in transition probabilities explains why the choice of $D_{0}=D_{3}$ yields closed-loop stability on average, while the one of $D_{0}=D_{4}$ does not. The prediction horizon should thus reflect on this distribution evolution to improve the compensation capabilities of the controller. Future works should focus on this aspect.

\section{Conclusion}

In this paper, we proposed a constant horizon predictionbased controller to compensate for a stochastic input delay modelled as a Markov process with a finite number of values. We proved the exponential mean-square stability of the closed-loop control system provided that the delay values are limited and in the vicinity of the chosen prediction horizon. Simulations on a toy example illustrated the relevance of this condition and the interest of this prediction-based control law.

Simulation results emphasize the crucial role played by the delay distribution and its evolution. However, the robustness analysis provided in this paper does not distinguish between the probability distributions. It is built on the worstcase scenario of a uniform probability of taking any delay value. Hence, the sufficient condition we obtain is likely to be somehow conservative. Relaxing this condition by including the delay distribution into the stability analysis is an important direction of future work. Adapting the predictionhorizon to the current delay distribution could also be an interesting design feature to explore, as it is likely to increase the closed-loop delay-robustness. Similarly, capturing the dependence on the feedback gain on this robustness margin is an important practical question for control tuning, which should be explored.

Finally, the delay process considered in this work has a finite number of states. Extending this analysis to the case where the delay can take values in a given continuum is another challenging theoretical issue worth exploring in the future. 


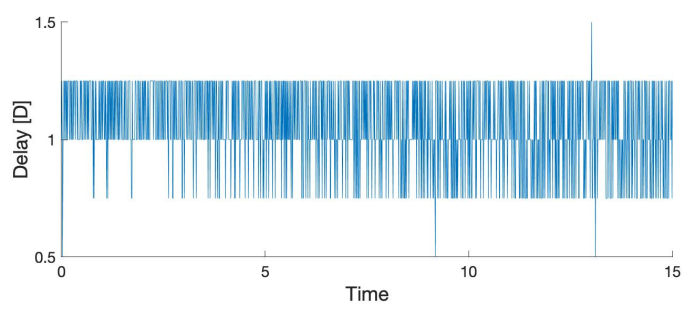

(a) Example of a realization of the stochastic delay $D$

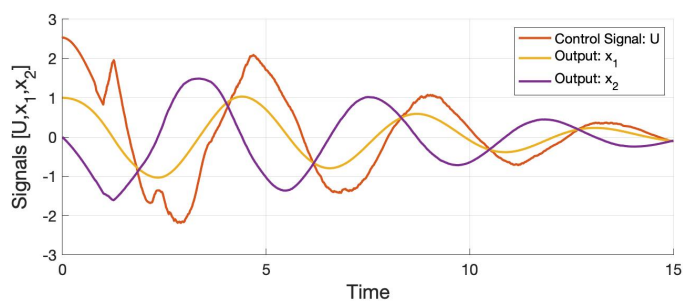

(b) Realization of the signals $U, x_{1}$ and $x_{2}$ corresponding to the delay pictured in (a)

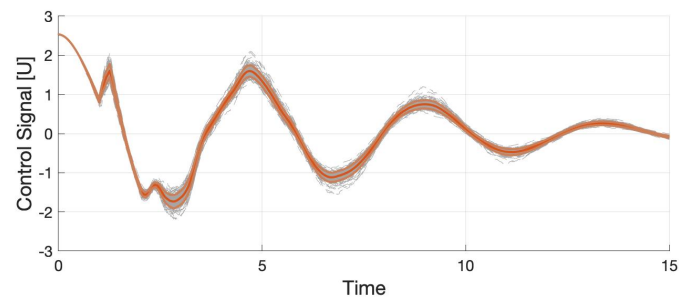

(c) Monte Carlo simulation of the closed-loop input $U$ (100 trials)

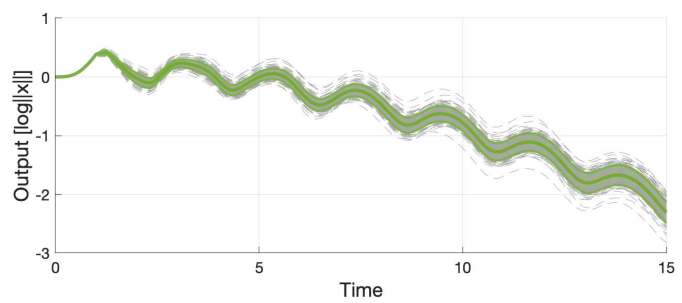

(d) Monte Carlo simulation of $\log \|x\|$ (100 trials)

Fig. 1. Simulation results of the closed-loop system (37) and (5) for $\mathbf{D}=(0.5,0.75,1.0,1.25,1.5)^{T}, X(0)=\left[\begin{array}{ll}1 & 0\end{array}\right]^{T}$ and $U(t)=0$ for $t \leq 0$. The prediction horizon is $D_{0}=1.0$. The transition probabilities follow the dynamics (38)-(39). (a) and (b) picture results corresponding to one delay realization. (c) and (d) present the results of 100 trials, in which the means and the standard deviations are highlighted by the coloured lines.

\section{A Technical Lemmas}

Lemma 5 Consider (3). There exists a constant $R_{0}$ such that the function $\Upsilon$ defined in (8) satisfies

$$
\Upsilon(t) \leq R_{0} \Upsilon(0), \quad t \in[0, \bar{D}]
$$

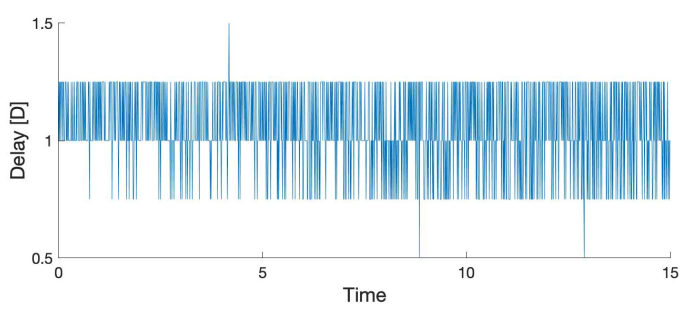

(a) Example of a realization of the stochastic delay $D$

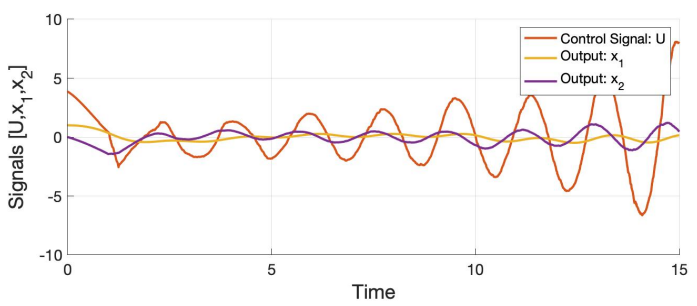

(b) Realization of the signals $U, x_{1}$ and $x_{2}$ corresponding to the delay pictured in (a)

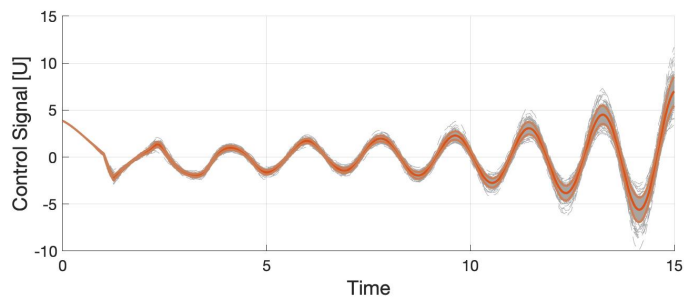

(c) Monte Carlo simulation of the closed-loop input $U$ (100 trials)

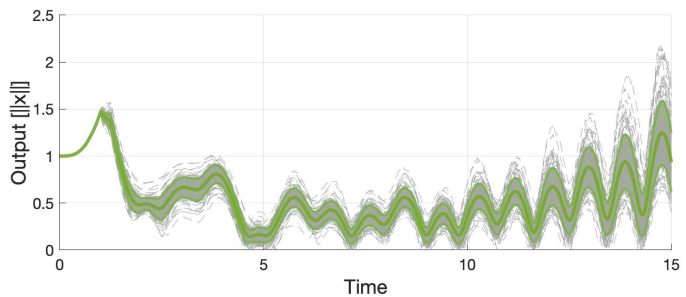

(d) Monte Carlo simulation of $\|x\|$ (100 trials)

Fig. 2. Simulation results of the closed-loop system (37) and (5) for $\mathbf{D}=(0.5,0.75,1.0,1.25,1.5)^{T}, X(0)=\left[\begin{array}{ll}1 & 0\end{array}\right]^{T}$ and $U(t)=0$ for $t \leq 0$. The prediction horizon is $D_{0}=1.25$. The transition probabilities follow the dynamics (38)-(39). (a) and (b) picture results corresponding to one delay realization. (c) and (d) present the results of 100 trials, in which the means and the standard deviations are highlighted by the coloured lines.

PROOF. Using (20) in Lemma 3, for $t \in[0, \bar{D}]$, and defining 
$N_{1}=2 e^{2|A| \bar{D}} \max \left\{1,|B|^{2}\right\}$, it holds

$$
\begin{aligned}
& |X(t)|^{2} \\
\leq & N_{1}\left(|X(0)|^{2}+\int_{-\bar{D}}^{t-\underline{D}} U(s)^{2} d s\right) \\
= & N_{1}\left(|X(0)|^{2}+\int_{-\bar{D}}^{\min \{t-\underline{D}, 0\}} U_{0}(s)^{2} d s+\int_{\min \{t-\underline{D}, 0\}}^{t-\underline{D}} U(s)^{2} d s\right)
\end{aligned}
$$

with $U(t)=U_{0}(t)$ for $t \leq 0$. Thus, by using Theorem 2 in [5], the prediction-based control law can be also written as

$U(t)=K_{D}\left[X(t)+\int_{0}^{t} \Phi_{D}(t, s) X(s) d s+\int_{-D_{0}}^{0} \Phi_{0}(t, s) U_{0}(s) d s\right]$

with $K_{D}=K e^{A D_{0}}$, and $\Phi_{D}$ and $\Phi_{0}$ two continuous functions defined in [5]. Replacing (A.3) into (A.2), one obtains

$$
\begin{aligned}
|X(t)|^{2} \leq & N_{1}\left(|X(0)|^{2}+\int_{-\bar{D}}^{\min \{t-\underline{D}, 0\}} U_{0}(s)^{2} d s\right. \\
& +3 K_{D} \int_{\min \{t-\underline{D}, 0\}}^{t-\underline{D}}|X(s)|^{2} d s \\
& +3 K_{D} \int_{\min \{t-\underline{D}, 0\}}^{t-\underline{D}} \int_{-D_{0}}^{0} \Phi_{0}(s, \xi)^{2} U_{0}(\xi)^{2} d \xi d s \\
& \left.+3 K_{D} \int_{\min \{t-\underline{D}, 0\}}^{t-\underline{D}} \int_{0}^{s} \Phi_{D}(s, \xi)^{2}|X(\xi)|^{2} d \xi d s\right)
\end{aligned}
$$

Thus, using again (A.2) and (A.3) and by a straightforward iteration on time intervals of length $\underline{D}$, we can get that there exist $N_{2}>0$ and a continuous function $\tilde{\Phi}_{0}$ such that

$$
|X(t)|^{2} \leq N_{2}|X(0)|^{2}+\int_{-\bar{D}}^{0} \tilde{\Phi}_{0}(t, s) U_{0}(s)^{2} d s
$$

Similarly, there exist a constant $N_{3}>0$ and a continuous function $\tilde{\Phi}_{0}$ such that

$$
U(t)^{2} \leq N_{3}|X(0)|^{2}+\int_{-\bar{D}}^{0} \tilde{\tilde{\Phi}}_{0}(t, s) U_{0}(s)^{2} d s
$$

Therefore, as from (8), it holds

$$
\Upsilon(t)=|X(t)|^{2}+\int_{t-\bar{D}-D_{0}}^{0} U_{0}(s)^{2} d s+\int_{0}^{t} U(s)^{2} d s
$$

the conclusion follows from (A.5) and (A.6).

Lemma 6 Consider the function $h$ defined in (16). There exists $M>0$ such that

$$
\left\|h\left(t+D_{0}(x-1)\right)\right\|^{2} \leq M V(t), \quad t+D_{0}(x-1) \geq 0
$$

PROOF. First, from (16), $h$ can be expressed as

$$
\begin{aligned}
h(t)= & D_{0} K\left[e^{A D_{0}} A X(t)+e^{A D_{0}} B U(t-D(t))+B U(t)\right. \\
& \left.-e^{A D_{0}} B U\left(t-D_{0}\right)+A \int_{t-D_{0}}^{t} e^{A(t-s)} B U(s) d s\right]
\end{aligned}
$$

Then, (A.9) gives

$$
\begin{aligned}
& \left\|h\left(t+D_{0}(x-1)\right)\right\|^{2} \\
= & \int_{0}^{1} h\left(t+D_{0}(x-1)\right)^{2} d x \\
\leq & 5|K|^{2} D_{0}^{2} \int_{0}^{1}\left[M_{1}\left|X\left(t+D_{0}(x-1)\right)\right|^{2}\right. \\
& +M_{2}\left|U\left(t+D_{0}(x-1)-D\left(t+D_{0}(x-1)\right)\right)\right|^{2} \\
& +M_{3}\left|U\left(t+D_{0}(x-1)\right)\right|^{2}+M_{4}\left|U\left(t+D_{0}(x-2)\right)\right|^{2} \\
& \left.+|A|^{2} \int_{t+D_{0}(x-2)}^{t+D_{0}(x-1)} e^{2|A|\left(t+D_{0}(x-1)-s\right)}|B|^{2}|U(s)|^{2} d s\right] d x
\end{aligned}
$$

with

$$
\left\{\begin{array}{l}
M_{1}=e^{2|A| \bar{D}}|A|^{2} \\
M_{2}=e^{2|A| \bar{D}}|B|^{2} \\
M_{3}=|B|^{2} \\
M_{4}=e^{2|A| \bar{D}}|B|^{2}
\end{array}\right.
$$

From the definition of the dynamics (3), it holds

$$
\begin{aligned}
& \left|X\left(t+D_{0}(x-1)\right)\right| \\
= & \left|e^{-A D_{0}(x-1)}\left(X(t)-\int_{t+D_{0}(x-1)}^{t} e^{A(t-s)} U(s-D(s)) d s\right)\right| \\
\leq & e^{|A| D_{0}}\left(|X(t)|+\int_{t+D_{0}(x-1)}^{t} e^{|A|(t-s)} \sum_{j=1}^{r}\left|U\left(s-D_{j}\right)\right| d s\right) \\
\leq & e^{|A| D_{0}}|X(t)|+e^{2|A| D_{0}} \sum_{j=1}^{r} \int_{t+D_{0}(x-1)}^{t}\left|U\left(s-D_{j}\right)\right| d s
\end{aligned}
$$

Then, with (19), the equation (A.10) gives

$$
\begin{aligned}
& \left\|h\left(t+D_{0}(x-1)\right)\right\|^{2} \\
\leq & 5|K|^{2} D_{0}^{2}\left[2 M_{1} e^{2|A| D_{0}}|X(t)|^{2}\right. \\
& +2 M_{1} r e^{4|A| D_{0}}\left(\|\mu(t)\|^{2}+M_{6}|X(t)|^{2}+M_{6}\|w(t)\|^{2}\right) \\
& +M_{2} r\left(\|\mu(t)\|^{2}+M_{6}|X(t)|^{2}+M_{6}\|w(t)\|^{2}\right) \\
& +M_{3} M_{6}|X(t)|^{2}+M_{3} M_{6}\|w(t)\|^{2}+M_{4}\|\mu(t)\|^{2} \\
& \left.+M_{5}\left(\|\mu(t)\|^{2}+M_{6}|X(t)|^{2}+M_{6}\|w(t)\|^{2}\right)\right] \\
\leq & 5|K|^{2} \bar{D}^{2}\left[M_{X}|X(t)|^{2}+M_{w}\|w(t)\|^{2}+M_{\mu}\|\mu(t)\|^{2}\right]
\end{aligned}
$$


in which $M_{5}=|A|^{2} e^{2|A| D_{0}}|B|^{2}, M_{6}=3\left(1+|K|^{2} e^{2|A+B K| D_{0}}\right.$ $\left.\max \left\{1, D_{0}^{2}|B|^{2}\right\}\right)$ and the positive constants $\left(M_{X}, M_{w}, M_{\mu}\right)$ are defined as follows

$$
\left\{\begin{array}{l}
M_{X}=2 M_{1} e^{2|A| \bar{D}}+M_{6}\left(2 M_{1} r e^{4|A| \bar{D}}+M_{2} r+M_{3}+M_{5}\right) \\
M_{w}=M_{6}\left(2 M_{1} r e^{4|A| \bar{D}}+M_{2} r+M_{3}+M_{5}\right) \\
M_{\mu}=2 M_{1} r e^{4|A| \bar{D}}+M_{2} r+M_{4}+M_{5}
\end{array}\right.
$$

Consequently, from the definition of Lyapunov functional $V(t)$ in (24)

$$
\begin{aligned}
& \left\|h\left(t+D_{0}(x-1)\right)\right\|^{2} \\
\leq & 5|K|^{2} \bar{D}^{2} \max \left\{\frac{M_{X}}{\min (\lambda(P))}, \frac{M_{w}}{b \underline{D}}, \frac{M_{\mu}}{d \bar{D}}\right\} V(t)
\end{aligned}
$$

The lemma is then proved with the positive constant $M=$ $5|K|^{2} \bar{D}^{2} \max \left\{\frac{M_{X}}{\min (\lambda(P))}, \frac{M_{w}}{b \underline{D}}, \frac{M_{\mu}}{d \bar{D}}\right\}$.

\section{References}

[1] Z. Artstein. Linear systems with delayed controls: a reduction. IEEE Transactions on Automatic control, 27(4):869-879, 1982.

[2] N. Bekiaris-Liberis and M. Krstic. Nonlinear control under nonconstant delays. SIAM, 2013.

[3] N. Bekiaris-Liberis and M. Krstic. Robustness of nonlinear predictor feedback laws to time-and state-dependent delay perturbations. Automatica, 49(6):1576-1590, 2013.

[4] D. Bresch-Pietri and D. Del Vecchio. Estimation for decentralized safety control under communication delay and measurement uncertainty. Automatica, 62:292-303, 2015.

[5] D. Bresch-Pietri, C. Prieur, and E. Trélat. New formulation of predictors for finite-dimensional linear control systems with input delay. Systems \& Control Letters, 113:9-16, 2018.

[6] F. Cacace, A. Germani, C. Manes, and M. Papi. Predictor based output-feedback control of linear stochastic systems with large i/o delays. IEEE Transactions on Automatic Control, 2020.

[7] R. Carli and F. Bullo. Quantized coordination algorithms for rendezvous and deployment. SIAM Journal on Control and Optimization, 48(3):1251-1274, 2009.

[8] J.-Y. Choi and M. Krstic. Compensation of time-varying input delay for discrete-time nonlinear systems. International Journal of Robust and Nonlinear Control, 26(8):1755-1776, 2016.

[9] J. C. Cortés, L. Jódar, and L. Villafuerte. Random linear-quadratic mathematical models: computing explicit solutions and applications. Mathematics and Computers in Simulation, 79(7):2076-2090, 2009.

[10] E. B. Dynkin. Theory of Markov processes. Courier Corporation, 2012.

[11] F. Fagnani and S. Zampieri. Average consensus with packet drop communication. SIAM Journal on Control and Optimization, 48(1):102-133, 2009.

[12] V. Gupta. On the effect of stochastic delay on estimation. IEEE Transactions on Automatic Control, 56(9):2145-2150, 2011.

[13] V. Gupta, A. F. Dana, J. P. Hespanha, R. M. Murray, and B. Hassibi. Data transmission over networks for estimation and control. IEEE Transactions on Automatic Control, 54(8):1807-1819, 2009.
[14] M. R. Hafner, D. Cunningham, L. Caminiti, and D. Del Vecchio. Cooperative collision avoidance at intersections: Algorithms and experiments. IEEE Transactions on Intelligent Transportation Systems, 14(3):1162-1175, 2013.

[15] J. K. Hale, S. M. V. Lunel, L. S. Verduyn, and S. M. V. Lunel. Introduction to functional differential equations, volume 99. Springer Science \& Business Media, 1993.

[16] K. Ito and M. Nisio. On stationary solutions of a stochastic differential equation. J. Math. Kyoto Univ., 4(1):1-75, 1964.

[17] I. Karafyllis and M. Krstic. Delay-robustness of linear predictor feedback without restriction on delay rate. Automatica, 49(6):17611767, 2013.

[18] I. Karafyllis and M. Krstic. Predictor feedback for delay systems: Implementations and approximations. Springer, 2017.

[19] I. Kats and N. Krasovskii. On the stability of systems with random parameters. Journal of Applied Mathematics and Mechanics, 24(5):1225-1246, 1960.

[20] V. Kolmanovskii and A. Myshkis. Introduction to the theory and applications of functional differential equations, volume 463. Springer Science \& Business Media, 2013.

[21] I. Kolmanovsky and T. L. Maizenberg. Mean-square stability of nonlinear systems with time-varying, random delay. Stochastic analysis and Applications, 19(2):279-293, 2001.

[22] I. V. Kolmanovsky and T. L. Maizenberg. Optimal control of continuous-time linear systems with a time-varying, random delay. Systems \& Control Letters, 44(2):119-126, 2001.

[23] S. Kong and D. Bresch-Pietri. Constant time horizon predictionbased control for linear systems with time-varyig input delay. In in Proc. of the 2020 IFAC World Congress, to appear.

[24] M. Krstic and A. Smyshlyaev. Boundary control of PDEs: A course on backstepping designs. SIAM, 2008.

[25] W. Kwon and A. Pearson. Feedback stabilization of linear systems with delayed control. IEEE Transactions on Automatic control, 25(2):266-269, 1980.

[26] H. Lhachemi, C. Prieur, and R. Shorten. An LMI condition for the robustness of constant-delay linear predictor feedback with respect to uncertain time-varying input delays. Automatica, 109:108551, 2019.

[27] K. Li and X. Mu. Predictor-based ho leader-following consensus of stochastic multi-agent systems with random input delay. Optimal Control Applications and Methods, 2021.

[28] A. Manitius and A. Olbrot. Finite spectrum assignment problem for systems with delays. IEEE transactions on Automatic Control, 24(4):541-552, 1979.

[29] S.-E. A. Mohammed. Stochastic functional differential equations, volume 99. Pitman Advanced Publishing Program, 1984.

[30] T. Molnár, W. Qin, T. Insperger, and G. Orosz. Application of predictor feedback to compensate time delays in connected cruise control. IEEE Transactions on Intelligent Transportation Systems, 19(2):545-559, 2017.

[31] S. Mondié, S.-I. Niculescu, and J. J. Loiseau. Delay robustness of closed loop finite assignment for input delay systems. IFAC Proceedings Volumes, 34(23):207-212, 2001.

[32] M. T. Nihtila. Finite pole assignment for systems with time-varying input delays. In Proceedings of the 30th IEEE Conference on Decision and Control, pages 927-928. IEEE, 1991.

[33] Z. J. Palmor. Time-delay compensation-Smith predictor and its modifications. The Control Handbook, pages 224-237, 1996.

[34] M. Rausand and A. Hoyland. System reliability theory: models, statistical methods, and applications, volume 396. John Wiley \& Sons, 2003.

[35] S. Ross. Introduction to probability models. Academic press, 2014. 
[36] A. Selivanov and E. Fridman. Predictor-based networked control under uncertain transmission delays. Automatica, 70:101-108, 2016.

[37] D. Simon, A. Seuret, and O. Sename. Real-time control systems: feedback, scheduling and robustness. International Journal of Systems Science, 48(11):2368-2378, 2017.

[38] O. J. M. Smith. A controller to overcome dead time. ISA J., 6:28-33, 1959.

[39] R. Song and Q. Zhu. Stability of linear stochastic delay differential equations with infinite markovian switchings. International Journal of Robust and Nonlinear Control, 28(3):825-837, 2018.

[40] H. T. Sykora, M. Sadeghpour, J. I. Ge, D. Bachrathy, and G. Orosz. On the moment dynamics of stochastically delayed linear control systems. International Journal of Robust and Nonlinear Control, 30(18):8074-8097, 2020.

[41] E. Witrant. Stabilisation des systèmes commandés par réseaux. PhD thesis, 2005.

[42] L. Zhang and G. Orosz. Beyond-line-of-sight identification by using vehicle-to-vehicle communication. IEEE Transactions on Intelligent Transportation Systems, 19(6):1962-1972, 2017. 